\title{
Effect of Ezetimibe on Remnant-Like Particle Cholesterol in Subjects with Metabolic Syndrome
}

\author{
Kazuhiko Kotania, ${ }^{a}$ Naoki Sakane ${ }^{a}$ Nobuyuki Taniguchi ${ }^{b}$ \\ a Department of Preventive Medicine and Diabetes Education, Clinical Research Institute, National Hospital \\ Organization Kyoto Medical Center, Kyoto, and b Department of Clinical Laboratory Medicine, Jichi Medical \\ University, Shimotsuke, Japan
}

\section{Key Words}

Remnant-like particle cholesterol • Remnant metabolism • Intestinal cholesterol transporter inhibitor · Atherosclerosis cant after adjusting for confounding factors. Conclusion: Ezetimibe monotherapy may be associated with a greater reduction in RLP-C levels in subjects with MetS than in those without MetS.

Copyright $\odot 2011$ S. Karger AG, Basel

\begin{abstract}
Objective: To investigate the influence of ezetimibe monotherapy on remnant-like particle cholesterol (RLP-C) in subjects with metabolic syndrome (MetS). Materials and Methods: Ezetimibe (10 mg/daily) was prescribed over a 12-week period for hypercholesterolemic subjects divided into groups with MetS ( $n=28$; male/female =13/15; mean age 67 years) and without MetS ( $n=22$; male/female $=9 / 13$; mean age 66 years). In the pre- and post-treatment phases, BMI, blood pressure and fasting blood levels of glucose, lipid panels and RLP-C were measured. Results: The group with MetS showed significantly higher RLP-C levels than the group without MetS [median level: 0.18 vs. $0.12 \mathrm{mmol} / \mathrm{l}$ (7.1 vs. 4.4 $\mathrm{mg} / \mathrm{dl}), \mathrm{p}<0.01$ ] in the pre-treatment phase. In the posttreatment phase, the low-density lipoprotein cholesterol levels were significantly reduced in both groups to a similar level ( $p<0.001$ in both), while there was a significantly greater reduction in RLP-C in the group with MetS than the group without MetS [median level: 0.12 vs. $0.11 \mathrm{mmol} / \mathrm{l}$ (4.8 vs. 4.1 $\mathrm{mg} / \mathrm{dl}), \mathrm{p}<0.05]$. This difference in RLP-C remained signifi-
\end{abstract}

\section{Introduction}

Metabolic syndrome (MetS) is a highly prevalent disorder related to high cardiovascular risk, which consists of multiple factors such as obesity, hypertension, low high-density lipoprotein cholesterol (HDL-C), high triglycerides (TG) and glucose intolerance [1-3]. While an increase in low-density lipoprotein cholesterol (LDL-C) in the circulation is recognized as a cardiovascular risk factor, remnant lipoproteins such as chylomicron and very low-density lipoprotein (VLDL) remnants have recently attracted attention, because remnant lipoproteins have been demonstrated to be associated with cardiovascular diseases [4-6]. Individuals with MetS frequently manifest such atherogenic lipid/lipoprotein profiles, including remnant-like particle cholesterol (RLP-C), measured as a reflection of remnant metabolism $[7,8]$. The increase in RLP-C in subjects with MetS may be ex-

\section{KARGER}

Fax +41613061234 E-Mail karger@karger.ch www.karger.com
(C) 2011 S. Karger AG, Basel

$1011-7571 / 12 / 0212-0134 \$ 38.00 / 0$

Accessible online at:

www.karger.com/mpp
Kazuhiko Kotani, MD, PhD

Department of Preventive Medicine and Diabetes Education, Clinical Research

Institute, National Hospital Organization Kyoto Medical Center

1-1 Fukakusa mukaihata, Fushimi-ku, Kyoto 612-8555 (Japan)

Tel. +81 756419 161, E-Mail kazukotani@jichi.ac.jp 
plained by several mechanisms: for example, an alteration of intestinallipid transport (lipid absorption/synthesis), intestinal overproduction of chylomicrons, hepatic overproduction of remnants, or a systemic clearance defect in remnants could all be involved [7, 9-13].

Ezetimibe was introduced as a new-concept agent, a selective inhibitor of intestinal cholesterol transporter, which suppresses the absorption of dietary and biliary cholesterol by inhibiting the Niemann-Pick-like 1 enterocyte receptor, thereby reducing circulating concentrations of LDL-C $[14,15]$. Because subjects with MetS can suffer from overproduction of chylomicrons in relation to intestinal lipid transport $[11,13,16]$, the ezetimibe treatment may reduce circulating levels of RLP-C, in addition to LDL-C, in subjects with MetS.

However, there is little clinical information available on the effect of ezetimibe monotherapy on RLP-C in subjects with MetS in particular, although one earlier report showed that combined ezetimibe-statin therapy reduced RLP-C levels in subjects with obesity and MetS [17]. In terms of the effects of ezetimibe monotherapy on nonMetS populations, some reports have shown a reduction in the RLP-C levels in subjects with dyslipidemia [18, 19], dyslipidemia and features of MetS [20], type 2 diabetes [21] and end-stage renal disease [22]. The present study was thus aimed to examine the influence of ezetimibe monotherapy on RLP-C in subjects with and without MetS.

\section{Subjects and Methods}

Ezetimibe (10 mg/daily) was prescribed as first-line treatment over a 12-week period for a total of 50 subjects with hypercholesterolemia [ $\geq 3.64 \mathrm{mmol} / \mathrm{l}(140 \mathrm{mg} / \mathrm{dl})$ of serum LDL-C concentrations]. These subjects were divided into two groups; those with MetS $(\mathrm{n}=28$; male/female $=13 / 15 ;$ mean age $=67.1 \pm 8.1$ years $)$ and those without MetS $(\mathrm{n}=22$; male/female $=9 / 13$; mean age $=$ $66.8 \pm 5.2$ years) based on their cardiometabolic characteristics in the pre-treatment phase. The eligible subjects had no history of cardiovascular, thyroid, kidney, liver or collagen disease. They included subjects with current smoking habits and without heavy alcohol intake (on self-reports). While the population did not receive any other lipid-lowering agents, those who were receiving anti-hypertensive agents (calcium inhibitors and/or angiotensinconverting enzyme inhibitors) and/or anti-diabetic agents (sulfonylureas) with fair diabetic control $(<7.5 \%$ of hemoglobin A1c; HbAlc) were included in the study. These agents were not changed during the treatment period. The study was approved by the institution's ethics committee and each subject gave informed consent.

The existence of 3 of 5 of the following criteria constituted a diagnosis of MetS according to the National Cholesterol Educa- tion Program Adult Treatment Panel (NCEP-ATP) III recommendations [23]: (1) obesity identified by a BMI $\geq 25.0 \mathrm{~kg} / \mathrm{m}^{2}$ as a surrogate for Japanese subjects [24]; (2) elevated blood pressure (BP) identified by systolic BP (SBP) $\geq 130 \mathrm{~mm} \mathrm{Hg}$ and/or diastolic $\mathrm{BP}(\mathrm{DBP}) \geq 85 \mathrm{~mm} \mathrm{Hg}$, or subjects who were receiving antihypertensive agents; (3) hypertriglyceridemia identified by serum TG $\geq 1.70 \mathrm{mmol} / \mathrm{l}$ (150 mg/dl); (4) decreased HDL-C identified by serum HDL-C $<1.03 \mathrm{mmol} / \mathrm{l}(40 \mathrm{mg} / \mathrm{dl})$ in males and $<1.29$ $\mathrm{mmol} / \mathrm{l}(50 \mathrm{mg} / \mathrm{dl})$ in females; (5) elevated glucose status identified by a fasting plasma glucose $\geq 6.1 \mathrm{mmol} / 1$ ( $110 \mathrm{mg} / \mathrm{dl})$, or subjects who were receiving anti-diabetic agents.

In the pre- and post-treatment phases, the following parameters were measured in the overnight fasting state: the BMI, SBP/ DBP, plasma glucose, HbAlc and serum LDL-C, TG, HDL-C and RLP-C levels. The concentrations of glucose, LDL-C, TG and HDL-C were measured by the standard enzymatic methods. HbAlc was measured by a high-performance liquid chromatographic method. Serum RLP-C was assayed by the homogeneous method, where cholesterol concentrations in chylomicron remnants, VLDL remnants and intermediate-density lipoproteins were measured with a polyoxyethylene-polyoxybutylene block copolymer and phospholipase-D in the presence of cholesterol esterase and cholesterol oxidase, as fully described in a previous report [25].

Unpaired t tests and $\chi^{2}$ tests were used to compare the above parameters in the pre-treatment phase between the groups with and without MetS. Paired t tests and McNemar tests were used to examine the pre- and post-treatment changes in the respective parameters within each group. A two-way (treatment period $\times$ group with and without MetS) ANOVA was used to compare the pre- and post-treatment changes in the RLP-C levels between the groups with and without MetS with adjustments for substantial confounding factors. TG and RLP-C were log-transformed because of their skewed distribution in the statistical analyses. Statistical significance was set at a value of $<0.05$.

\section{Results}

Among the variables in the pre-treatment phase, the levels of BMI, TG, glucose, HbAlc and RLP-C, in addition to the prevalence of smoking habits and prescription of anti-diabetic agents, were significantly higher, and the levels of HDL-C were significantly lower, in the group with MetS than in the group without MetS (table 1). In the post-treatment phase, 8 subjects with MetS did not meet the MetS diagnostic criteria in the group with MetS (normalization rate, $28.6 \%, \mathrm{p}=0.01$ ). In the post-treatment phase, the levels of LDL-C were significantly reduced from those of the pre-treatment phase within both groups to a similar level ( $\mathrm{p}<0.001$ in both groups, table 1). There was a significant reduction in RLP-C within the group with MetS $(p=0.005)$, while no significant reduction in RLP-C was observed within the group without $\operatorname{MetS}(p=0.418)$. 
Table 1. Pre- and post-treatment clinical characteristics of subjects with and without MetS

\begin{tabular}{|c|c|c|c|c|}
\hline & \multicolumn{2}{|c|}{ Without MetS $(\mathrm{n}=22)$} & \multicolumn{2}{|l|}{ With MetS $(\mathrm{n}=28)$} \\
\hline & pre & post & pre & post \\
\hline Male/female & $9 / 13$ & - & $13 / 15$ & - \\
\hline Age, years & $66.8 \pm 5.2$ & - & $67.1 \pm 8.1$ & - \\
\hline Smoking habit, $\%$ & $36^{*}$ & 36 & $64^{*}$ & 64 \\
\hline Anti-hypertensive agent, \% & 27 & 27 & 32 & 32 \\
\hline Anti-diabetic agent, $\%$ & $18^{* *}$ & 18 & $64^{* *}$ & 64 \\
\hline $\mathrm{BMI}, \mathrm{kg} / \mathrm{m}^{2}$ & $23.9 \pm 3.1^{* *}$ & $24.9 \pm 2.2$ & $27.6 \pm 3.9^{* *}$ & $25.9 \pm 4.0$ \\
\hline SBP, mm Hg & $135 \pm 16$ & $132 \pm 15$ & $136 \pm 17$ & $135 \pm 16$ \\
\hline $\mathrm{DBP}, \mathrm{mm} \mathrm{Hg}$ & $71 \pm 12$ & $74 \pm 8$ & $74 \pm 10$ & $73 \pm 11$ \\
\hline LDL-C, $\mathrm{mmol} / \mathrm{l}$ & $3.94 \pm 0.45$ & $3.16 \pm 0.50^{\dagger \dagger}$ & $3.97 \pm 0.52$ & $3.20 \pm 0.45^{\dagger \dagger}$ \\
\hline $\mathrm{mg} / \mathrm{dl}$ & $151.6 \pm 17.2$ & $121.5 \pm 19.3^{\dagger \dagger}$ & $152.6 \pm 20.1$ & $122.9 \pm 17.2^{\dagger \dagger}$ \\
\hline $\mathrm{HDL}-\mathrm{C}, \mathrm{mmol} / \mathrm{l}$ & $1.56 \pm 0.34^{*}$ & $1.57 \pm 0.38$ & $1.35 \pm 0.36^{*}$ & $1.35 \pm 0.41$ \\
\hline $\mathrm{mg} / \mathrm{dl}$ & $59.8 \pm 13.2^{*}$ & $60.4 \pm 14.7$ & $51.9 \pm 13.8^{*}$ & $52.0 \pm 15.8$ \\
\hline $\mathrm{TG}, \mathrm{mmol} / \mathrm{l}$ & $1.39(1.00-1.70)^{* *}$ & $1.46(0.99-1.59)$ & $1.78(1.39-2.41)^{* *}$ & $1.57(1.21-2.14)$ \\
\hline $\mathrm{mg} / \mathrm{dl}$ & $123(88-150)^{* *}$ & $129(88-142)$ & $157(123-213)^{* *}$ & $139(107-189)$ \\
\hline RLP-cholesterol, mmol/l & $0.12(0.09-0.18)^{* *}$ & $0.11(0.08-0.14)$ & $0.18(0.13-0.24)^{* *}$ & $0.12(0.09-0.18)^{\dagger \dagger}$ \\
\hline $\mathrm{mg} / \mathrm{dl}$ & $4.4(3.3-6.9)^{* *}$ & $4.1(3.1-5.3)$ & $7.1(5.0-9.2)^{* *}$ & $4.8(3.5-6.9)^{\dagger \dagger}$ \\
\hline Fasting blood glucose, $\mathrm{mmol} / \mathrm{l}$ & $6.49 \pm 1.80^{* *}$ & $6.51 \pm 1.83$ & $8.28 \pm 1.49^{* *}$ & $8.15 \pm 2.29$ \\
\hline $\mathrm{mg} / \mathrm{dl}$ & $116.9 \pm 32.4^{* *}$ & $117.2 \pm 32.9$ & $149.1 \pm 26.7^{* *}$ & $146.8 \pm 41.2$ \\
\hline HbAlc, \% & $5.6 \pm 0.9^{* *}$ & $5.7 \pm 1.0$ & $6.6 \pm 0.7^{* *}$ & $6.7 \pm 0.7$ \\
\hline
\end{tabular}

Data are presented as means \pm SD or medians (IQR), unless otherwise indicated. TG and RLP-C were log-transformed because of their skewed distributions. ${ }^{*} \mathrm{p}<0.05,{ }^{* *} \mathrm{p}<0.01$, pre-treatment data between the study groups (unpaired $\mathrm{t}$ test or $\chi^{2}$-test); ${ }^{\mathrm{t}} \mathrm{p}<0.01$, pre- vs. post-treatment data within each group (paired t test).

The change in RLP-C in the group with MetS was significantly greater than the change in the group without MetS ( $p=0.014)$. The difference in RLP-C between the two groups remained significant after adjusting for the following confounding factors: age, gender and smoking $(p=0.030)$; age, gender, smoking and pre-treatment LDL$\mathrm{C}(\mathrm{p}=0.031)$; age, gender, smoking, and the presence of anti-hypertensive agents and anti-diabetic agents $(\mathrm{p}=$ 0.013 ).

\section{Discussion}

In the present study, subjects with MetS exhibited more atherogenic characteristics, as seen in the levels of obesity and glucose/lipid panels plus the serum RLP-C level in the pre-treatment phase, compared to those without MetS. Given the pathophysiology of MetS [1-3], this is natural and a confirmation of the data observed in previous studies $[7,8]$. Of note, in this study using ezetimibe monotherapy, there was a significantly greater reduction in RLP-C in subjects with MetS in comparison to those without MetS, even though the glucose/lipid parameters of the MetS criteria did not remarkably change during the pre- and post-treatment phases. This finding appears to be meaningful when considering the clinical relevance of RLP-C in individuals with MetS with regard to cardiovascular diseases [4-6]. On the other hand, the effects of ezetimibe in clinical scenarios of cardiovascular diseases remain to be sufficiently proven compared to other lipidlowering agents such as statins, while some studies indicate that ezetimibe could improve endothelial function in certain populations such as those with MetS [26, 27]. There is an opinion that ezetimibe can be used when statins are not well tolerated or as an additional option when more than one LDL-C lowering is required [20]. Therefore, although the data of the present study suggest a positive use of ezetimibe for populations with MetS relative to those without MetS, more investigations are required to establish the usefulness of ezetimibe as an option for the lipid/lipoprotein management in MetS.

The biological mechanism underlying the present results remains unclear; however, several possibilities exist. For instance, ezetimibe, due to its nature as an inhibitor 
of intestinal cholesterol transporter, may attenuate the intestinal overproduction of chylomicrons, which has been suggested to exist in individuals with MetS $[11,13]$. A recent study in mice, which lack of hepatic Niemann-Picklike 1 expression and thereby have an advantage when trying to understand the lipid mechanism in small intestines, has clearly shown molecular mechanisms of ezetimibe-induced attenuation of postprandial hypertriglyceridemia by reducing the absorption of both cholesterol and long-chain fatty acids through enterocytes [16]. This can lead to the reduction in RLP-C via suppressed chylomicron remnants, and to the reduction of cholesterol inflow into the liver (subsequently, the remnant receptors of the liver can be up-regulated) [18]. Hepatic overproduction of remnants (VLDL in particular) is associated with hepatic steatosis [12], and ezetimibe ameliorates the hepatic steatosis and improves insulin resistance $[28,29]$. The improved insulin resistance can also improve intestinal lipid transport $[9,10,13]$, and beneficial hepatic changes will result in a further reduction in the RLP-C levels.

This study has some limitations. Although this was a prospective study, a randomized-controlled design was not applied. There were only a small number of participants, and they were all Japanese individuals. The data regarding intestinal cholesterol transport-related mark- ers, as well as genetic and residual lifestyle components, were not included in the study. Therefore, even though the present study seems to provide insight into daily lipid treatment in MetS, this study remains preliminary, and caution must be exercised in interpreting the current results.

\section{Conclusion}

The findings of the present study suggest that ezetimibe monotherapy may induce a greater reduction in the circulating RLP-C levels in subjects with MetS than in those without MetS. More studies are needed to confirm the present findings and to clarify the mechanisms underlying such a relationship.

\section{Acknowledgements}

The authors are grateful to Dr. Miyamoto and Dr. Okada for the assistance of this work. This study was supported in part by a Grant-in-Aid for the Scientific Research from the Ministry of education, Culture, Sports, Science, and Technology of Japan (K.K.) and the Foundation for the Development of the Community, Japan.

\section{References}

1 Gupta A, Gupta V: Metabolic syndrome: what are the risks for humans? Biosci Trends 2010;4:204-212.

-2 Mottillo S, Filion KB, Genest J, Joseph L, Pilote L, Poirier P, Rinfret S, Schiffrin EL, Eisenberg MJ: The metabolic syndrome and cardiovascular risk a systematic review and meta-analysis. J Am Coll Cardiol 2010;56: 1113-1132.

3 Dagogo-Jack S, Egbuonu N, Edeoga C: Principles and practice of nonpharmacological interventions to reduce cardiometabolic risk. Med Princ Pract 2010;19:167-175.

4 Nakamura T, Takano H, Umetani K, Kawabata K, Obata JE, Kitta Y, Kodama Y, Mende A, Ichigi Y, Fujioka D, Saito Y, Kugiyama K: Remnant lipoproteinemia is a risk factor for endothelial vasomotor dysfunction and coronary artery disease in metabolic syndrome. Atherosclerosis 2005;181:321-327.

$\checkmark 5$ Nakajima K, Nakano T, Tanaka A: The oxidative modification hypothesis of atherosclerosis: the comparison of atherogenic effects on oxidized LDL and remnant lipoproteins in plasma. Clin Chim Acta 2006;367: 36-47.

Ezetimibe, RLP-C and Metabolic

Syndrome
-6 Nakamura T, Obata JE, Takano H, Kawabata K, Sano K, Kobayashi T, Fujioka D, Saito Y, Yano T, Kugiyama K: High serum levels of remnant lipoproteins predict ischemic stroke in patients with metabolic syndrome and mild carotid atherosclerosis. Atherosclerosis 2009;202:234-240.

7 Chan DC, Watts GF, Barrett PH, O’Neill FH Redgrave TG, Thompson GR: Relationships between cholesterol homoeostasis and triacylglycerol-rich lipoprotein remnant metabolism in the metabolic syndrome. Clin Sci 2003;104:383-388.

$>8$ Satoh A, Adachi H, Tsuruta M, Hirai Y, Hiratsuka A, Enomoto M, Furuki K, Hino A, Takeuchi T, Imaizumi T: High plasma level of remnant-like particle cholesterol in the metabolic syndrome. Diabetes Care 2005;28: 2514-2518.

9 Gylling H, Miettinen TA: Cholesterol absorption: influence of body weight and the role of plant sterols. Curr Atheroscler Rep 2005;7:466-471.
10 Hoenig MR, Kostner KM, Read SJ, Walker PJ, Atherton JJ: Implications of the obesity epidemic for statin therapy: shifting cholesterol metabolism to a high synthesis and low dietary absorption state. Endocr Metab Immune Disord Drug Targets 2007;7:153166.

11 Vine DF, Takechi R, Russell JC, Proctor SD: Impaired postprandial apolipoprotein-B48 metabolism in the obese, insulin-resistant JCR:LA-cp rat: increased atherogenicity for the metabolic syndrome. Atherosclerosis 2007;190:282-290.

12 Adiels M, Olofsson SO, Taskinen MR, Borén J: Overproduction of very low-density lipoproteins is the hallmark of the dyslipidemia in the metabolic syndrome. Arterioscler Thromb Vasc Biol 2008;28:1225-1236.

13 Vine DF, Glimm DR, Proctor SD: Intestinal lipid transport and chylomicron production: possible links to exacerbated atherogenesis in a rodent model of the metabolic syndrome. Atheroscler Suppl 2008;9:69-76. 
-14 Lioudaki E, Ganotakis ES, Mikhailidis DP: Ezetimibe; more than a low density lipoprotein cholesterol lowering drug? An update after 4 years. Curr Vasc Pharmacol 2011;9: 62-86.

15 Betters JL, Yu L: NPC1L1 and cholesterol transport. FEBS Lett 2010;584:2740-2747.

-16 Sandoval JC, Nakagawa-Toyama Y, Masuda D, Tochino Y, Nakaoka H, Kawase R, YuasaKawase M, Nakatani K, Inagaki M, Tsubakio-Yamamoto K, Ohama T, Matsuyama A, Nishida M, Ishigami M, Komuro I, Yamashita S: Molecular mechanisms of ezetimibe-induced attenuation of postprandial hypertriglyceridemia. J Atheroscler Thromb 2010;17:914-924.

-17 Hajer GR, Dallinga-Thie GM, van Vark-van der Zee LC, Visseren FL: The effect of statin alone or in combination with ezetimibe on postprandial lipoprotein composition in obese metabolic syndrome patients. Atherosclerosis 2009;202:216-224.

- 18 Masuda D, Nakagawa-Toyama Y, Nakatani $\mathrm{K}$, Inagaki M, Tsubakio-Yamamoto K, Sandoval JC, Ohama T, Nishida M, Ishigami M, Yamashita S: Ezetimibe improves postprandial hyperlipidaemia in patients with type IIb hyperlipidaemia. Eur J Clin Invest 2009; 39:689-698.
19 Miyashita Y, Endo K, Saiki A, Ban N, Nagumo A, Yamaguchi T, Kawana H, Nagayama D, Ohira M, Oyama T, Shirai K: Effect of ezetimibe monotherapy on lipid metabolism and arterial stiffness assessed by cardio-ankle vascular index in type 2 diabetic patients. J Atheroscler Thromb 2010;17:1070-1076.

20 Ansquer JC, Bekaert I, Guy M, Hanefeld M, Simon A; Study Investigators: Efficacy and safety of coadministration of fenofibrate and ezetimibe compared with each as monotherapy in patients with type IIb dyslipidemia and features of the metabolic syndrome: a prospective, randomized, double-blind, three-parallel arm, multicenter, comparative study. Am J Cardiovasc Drugs 2009;9:91101.

21 Nozue T, Michishita I, Mizuguchi I: Effects of ezetimibe on remnant-like particle cholesterol, lipoprotein (a), and oxidized lowdensity lipoprotein in patients with dyslipidemia. J Atheroscler Thromb 2010;17:37-44.

22 Hirano T, Nohtomi K, Nakanishi N, Watanabe T, Hyodo T, Taira T: Ezetimibe decreases serum amyloid A levels in HDL3 in hemodialysis patients. Clin Nephrol 2010;74:282287.

23 Expert Panel on detection, Evaluation, and Treatment of High Blood Cholesterol in Adults: Executive summary of the third report of the National Cholesterol Education Program (NCEP) expert panel on detection, evaluation, and treatment of high blood cholesterol in adults (Adult Treatment Panel III) JAMA 2001;285:2486-2497.
24 Examination Committee of Criteria for 'Obesity Disease' in Japan; Japan Society for the Study of Obesity: New criteria for 'obesity disease' in Japan. Circ J 2002;66:987992.

25 Miyauchi K, Kayahara N, Ishigami M, Kuwata H, Mori H, Sugiuchi H, Irie T, Tanaka A, Yamashita S, Yamamura T: Development of a homogeneous assay to measure remnant lipoprotein cholesterol. Clin Chem 2007;53: 2128-2135.

-26 Tenenbaum A, Fisman EZ, Motro M, Adler Y: Atherogenic dyslipidemia in metabolic syndrome and type 2 diabetes: therapeutic options beyond statins. Cardiovasc Diabetol 2006;5:20.

$\checkmark 27$ Bass A, Hinderliter AL, Lee CR: The impact of ezetimibe on endothelial function and other markers of cardiovascular risk. Ann Pharmacother 2009;43:2021-2030.

-28 Deushi M, Nomura M, Kawakami A, Haraguchi $\mathrm{M}$, Ito $\mathrm{M}$, Okazaki M, Ishii $\mathrm{H}$, Yoshida $\mathrm{M}$ : Ezetimibe improves liver steatosis and insulin resistance in obese rat model of metabolic syndrome. FEBS Lett 2007;581:56645670

-29 Fukuda M, Nakamura T, Kataoka K, Nako H, Tokutomi Y, Dong YF, Yasuda O, Ogawa $\mathrm{H}$, Kim-Mitsuyama S: Ezetimibe ameliorates cardiovascular complications and hepatic steatosis in obese and type 2 diabetic $\mathrm{db} / \mathrm{db}$ mice. J Pharmacol Exp Ther 2010;335:70-75. 